\title{
The Effect of Selective Retina Therapy for Bevacizumab-Resistant Chronic Central Serous Chorioretinopathy
}

\author{
Seung Hee Jeon Minhee Kim Jiyoung Lee Young-Jung Roh \\ Department of Ophthalmology and Visual Science, Catholic University of Korea, Seoul, Republic of Korea
}

\section{Keywords}

Bevacizumab-resistant - Chronic central serous chorioretinopathy $\cdot$ Real-time feedback-controlled dosimetry · Retinal pigment epithelium - Selective retina therapy

\begin{abstract}
Purpose: The aim of this study was to evaluate the efficacy of selective retina therapy (SRT), used in conjunction with real-time feedback dosimetry (RFD), in the treatment of bevacizumab-resistant chronic central serous chorioretinopathy (CSC). Patients and Methods: In this retrospective cohort study, 22 eyes of 22 patients with bevacizumab-resistant chronic CSC, showing focal or diffuse foveal leakages on fundus fluorescein angiography (FFA), were included. After evaluation of the test spots at temporal arcades, SRT (wavelength, $527 \mathrm{~nm}$; pulse repetition rate, $100 \mathrm{~Hz}$; ramping over maximal 15 micropulses; and spot diameter, $200 \mu \mathrm{m}$ ) using RFD was applied to the leakage sites observed on FFA. Changes in the mean best-corrected visual acuity (BCVA), central macular thickness (CMT), and subretinal fluid (SRF) height were evaluated at baseline and at 1, 3, 6, 9, and 12 months following treatment. Results: SRF completely re-
\end{abstract}

\begin{tabular}{ll}
\hline karger@karger.com & (c) 2021 The Author(s). \\
Pww.karger.com/oph & $\begin{array}{l}\text { Published by S. Karger AG, Basel } \\
\text { This is an Open Access article licensed under the Creative Commons }\end{array}$ \\
Karger & $\begin{array}{l}\text { Attribution-NonCommercial-4.0 International License (CC BY-NC) } \\
\text { (http://www.karger.com/Services/OpenAccessLicense), applicable to } \\
\text { the online version of the article only. Usage and distribution for com- } \\
\text { mercial purposes requires written permission. }\end{array}$
\end{tabular}

solved in $81.8 \%$ (18/22 eyes) cases at 12 months post-treatment. The mean BCVA (logarithm of the minimum angle of resolution [logMAR]) improved from $0.49 \pm 0.29$ at baseline to $0.43 \pm 0.36$ at 12 months ( $p=0.067$ ). The mean BCVA gain was 0.06 logMAR, equivalent to 3 ETDRS letters. The CMT significantly decreased from $323 \pm 85.6 \mu \mathrm{m}$ at baseline to 221.5 $\pm 60.4 \mu \mathrm{m}$ at 12 months $(p<0.001)$. The mean SRF height also significantly decreased from $174.6 \pm 86.4 \mu \mathrm{m}$ at baseline to $35.1 \pm 75.4 \mu \mathrm{m}$ at 12 months $(p<0.001)$. Conclusion: SRT showed favorable visual and anatomical outcomes in patients with bevacizumab-resistant chronic CSC.

๑) 2021 The Author(s).
Published by S. Karger AG, Basel

\section{Introduction}

Central serous chorioretinopathy (CSC) is a chorioretinal disease that is characterized by idiopathic serous detachment of the neurosensory retina, with or without coexisting pigment epithelial detachment (PED) [1]. The incidence of CSC is estimated at 1 per 10,000, affecting middle-aged men predominantly [2].

Although the pathogenesis of CSC remains poorly understood, choroidal hyperpermeability with increased 
hydrostatic pressure induces the formation of PED and overpowers the barrier function of the retinal pigment epithelium (RPE), leading to subretinal fluid (SRF) accumulation [2,3]. Choroidal hyperpermeability is presumably related to abnormal choroidal circulation, as demonstrated by indocyanine green angiography (ICG) and swept-source optical coherence tomography (OCT) angiography $[4,5]$. The breakdown of the RPE monolayer, the so-called outer blood-retina barrier, is also thought to result in SRF accumulation, leading to acute CSC [6]. In acute CSC, the SRF can spontaneously resolve within 3-4 months, with good visual function outcomes, whereas chronic CSC with SRF persisting for longer than 3-6 months is often associated with diffuse RPE atrophic changes, resulting in irreversible photoreceptor damage and poor visual outcomes [7-10]. Therefore, complete elimination of SRF is the goal of CSC treatment $[11,12]$.

Although several treatment strategies, including conventional laser photocoagulation, photodynamic therapy (PDT) using verteporfin, and intravitreal anti-vascular endothelial growth factor (anti-VEGF) agents have been used for CSC, no standard treatment exists currently [12]. Furthermore, each treatment is associated with several ocular and systemic complications. Adverse events such as central scotoma and choroidal neovascularization $(\mathrm{CNV})$ have been reported following conventional laser photocoagulation $[8,13,14]$. Although anti-VEGF has been investigated as CSC treatment in several studies, its use for CSC is still off-label $[15,16]$. Despite PDT showing favorable clinical outcomes in previous studies, acute vision loss owing to RPE atrophy has been reported following full-dose or reduced-dose PDT $[8,17,18]$.

To avoid photoreceptor damage from conventional laser photocoagulation, selective retina therapy (SRT), known as photoreceptor-sparing micropulse laser (1.7 $\mu \mathrm{s})$, has been developed. Since SRT induces RPE rejuvenation in the SRT-treated area by stimulating RPE migration and proliferation, without affecting the adjacent neurosensory retina, it might be advantageous for treating macular disease such as CSC [19].

In previous studies, SRT showed favorable functional and anatomical outcomes for CSC treatment, and 2 clinical endpoints, including "invisible on color fundus photography (CFP)" and "visible on fundus fluorescein angiography (FFA)," have been used to titrate pulse energy for selective RPE damage [20-25]. To titrate the adequate pulse energy instantly without using FFA, SRT device employed with real-time feedback-controlled dosimetry (RFD) was developed. Briefly, when one of the micropulses between the 1st and 15th micropulses induces mi- crovaporization at the melanosome of RPE cells, RFD detects short-lived microbubbles causing RPE cell damage by using both optoacoustic dosimetry and reflectometry. The optoacoustic dosimetry detects ultrasonic pressure wave signals from the microbubbles, producing an optoacoustic feedback value $[19,26]$, whereas reflectometry detects modulations of backscattered light signals from the microbubbles, producing an optical feedback value [27-29]. RFD has been optimized in animal and clinical studies [23-25, 28, 30, 31].

Since persistent SRF can lead to severe vision loss due to irreversible photoreceptor damage [11], complete resolution of SRF is essential for restoring normal functional and anatomical interactions between photoreceptors and RPE. Although anti-VEGF agents have been used for treating patients with chronic CRC, other treatment modalities should be considered if CSC is refractory to repeated intravitreal anti-VEGF injections. While previous studies have investigated the use of SRT for CSC, herein, we first evaluated the effect of SRT used in conjunction with RFD in the treatment of bevacizumab-resistant chronic CSC.

\section{Materials and Methods}

The medical records of 26 patients who had undergone SRT with RFD for the treatment of chronic CSC who were previously treated with $\geq 3$ intravitreal consecutive bevacizumab injections during the period from January 2015 to February 2019 were reviewed. This retrospective study adhered to the principles of the Declaration of Helsinki, and data collection was compliant with and approved by the Institutional Review Board of the hospital, Korea. Written informed consent was obtained from all the patients in this study following an explanation of the possible risks of SRT treatment including retinal burns, retinal hemorrhages, and central scotoma.

Our inclusion criteria were as follows: (1) the presence of SRF involving the fovea in the OCT images for a period of 6 months or longer, despite at least 3 consecutive off-label use of $1.25 \mathrm{mg}$ intravitreal bevacizumab injections administered for $>4$ and up to 8 months, (2) availability of at least 12 months of medical records following SRT, and (3) presence of focal or diffuse leakage on FFA owing to CSC. If the number of pinpoint leakages on FFA was $\leq 3$, it was classified as focal type. Four or more leakages were classified as diffuse type. The exclusion criteria were as follows: (1) presence of other retinal diseases, including age-related macular degeneration, polypoidal choroidal vasculopathy, and pathological myopia; (2) presence of retinal exudation and hemorrhage indicating CNV; (3) a history of undergoing conventional laser and PDT before inclusion; and (4) anti-VEGF therapy within 3 months before inclusion.

To evaluate the response to SRT for CSC, all the patients underwent complete ophthalmological examination, including slitlamp evaluation and best-corrected visual acuity (BCVA) at base-
92

Ophthalmologica 2022;245:91-100 DOI: $10.1159 / 000520187$
Jeon/Kim/Lee/Roh 
line, and at 1, 3, 6, 9, and 12 months following treatment. Visual acuity was estimated using a standard Snellen chart and was converted to the logarithm of the minimum angle of resolution (logMAR). Multimodal imaging studies at baseline were performed in all patients after pupil dilatation. These included CFP (CF-60UVi; Canon Inc., Ota, Japan), FFA (HRA2; Heidelberg Engineering, Dosenheim, Germany), ICG (HRA2), and fundus autofluorescence (FAF) (HRA2; Heidelberg Engineering). In addition, spectral domain OCT (Cirrus; Carl Zeiss Meditec, Dublin, CA, USA) was used to detect the presence of SRF and measure the central macular thickness (CMT) and SRF height (maximum distance between the outer neurosensory retina and the RPE) using the macular cube scan protocol (central $6 \times 6 \mathrm{~mm}^{2}$ area, $512 \times 128 \mathrm{scan}$ ). The CFP, FAF, and OCT images were taken at baseline and at each visit. At the treatment day (within 2 weeks after performing the baseline examinations), FFA, CFP, and FAF were performed again $1 \mathrm{~h}$ after test spot irradiation, to determine the preset pulse energy for treatment spots. The SRF height was measured by 2 independent retinal specialists. Based on the SRF resolution rate after SRT, the responses to SRT were classified into 3 groups: complete resolution, $\geq 50 \%$ reduction, and $<50 \%$ reduction of SRF height were classified as "complete response," "partial response," and "no response," respectively.

\section{SRT Procedure}

A single surgeon (YJR) performed all the SRT using the SRT laser system (R:GEN; Lutronic, Goyang-si, South Korea), a neodymium-doped yttrium lithium fluoride-laser at a wavelength of $527 \mathrm{~nm}$, a maximum of 15 micropulses per spot, and a single micropulse duration of $1.7 \mu$ s, with a pulse repetition rate of $100 \mathrm{~Hz}$ and spot diameter of $200 \mu \mathrm{m}$. The SRT system is a device with $\mathrm{RFD}$, and the system is equipped with a photodiode as a reflectometric sensor and a specifically designed contact lens (field-ofview, $90 \mathrm{D}$; image magnification $\times 1.05$ ) with an inserted ultrasonic transducer as an optoacoustic sensor (Lutronic). The SRT device received a CE mark from the EU Notified Body and was approved for CSC treatment by the Ministry of Food and Drug Safety in South Korea.

In the current study, although RFD was used, exposure evaluation test spots were performed around the temporal arcade vessels before irradiating treatment spots in order to determine the individual optimal preset pulse energy, as previously described [20-25]. As adequate SRT spots were invisible on CFP, the minimum pulse energy among the FFA-positive spots was chosen as the initial treatment spot. SRT spots were applied to cover the area of foveal leakage on FFA. Since the SRT spots were invisible during irradiation, a 1-spot spacing of the SRT spots was left using a guide beam. Although RFD was used in the current study, the safety margin of the preset pulse energy was obtained based on angiographic features and CFP image. The upper limit was a minimum pulse energy showing a CFP-positive and FFA-positive spot, and the lower limit was a minimum pulse energy showing a FFA-positive and CFP-negative spot. All preset pulse energy of irradiations were planned to be within the safety margin.

The RFD was set with fixed thresholds, comprising 2.0 arbitrary units for the optoacoustic sensor and 6.0 arbitrary units for the reflectometric sensor. When one of the 15 ramping micropulses of each irradiation produces microbubbles at RPE cells, both optoacoustic and reflectometric values originated from microbubbles are displayed by the RFD. Whether the optoacoustic or reflec-

SRT for Bevacizumab-Resistant Chronic Central Serous Chorioretinopathy tometric value reaches the threshold, the RFD provides 3 types of arrow signals based on the placement of microbubble between the 1 st and 15th micropulse. According to the feedback signals of the RFD, the preset pulse energy indicating the possible 15th micropulse energy of each SRT spot was adjusted by the surgeon, based on the response to the previous irradiation. The side-pointing arrow indicating adequate treatment demands no adjustment. Furthermore, if the downward-pointing arrow (overtreatment alarm) appeared as a feedback response, the preset energy was decreased by $10 \mu \mathrm{J}$, and for the upward-pointing arrow (undertreatment alarm), the preset energy was increased by $10 \mu \mathrm{J}$, as previously described $[23,24,31]$.

If the SRF persisted on OCT by 3 months following SRT, additional SRT was performed using the same initial preset pulse energy that was used as initial irradiation in the 1st SRT session. Additional SRT treatments were applied to the same leakage area as in the initial presentation. Repeated FFA examination was planned if CNV was suspected on OCT during the follow-up period.

\section{Statistical Analysis}

The change in logMAR BCVA, CMT, and SRF height from baseline to the 1-, 3-, 6-, 9-, and 12-month visits was analyzed using paired $t$ tests. All values are presented as the mean \pm standard deviation (SD). A $p$ value of $<0.05$ was considered statistically significant. Statistical analyses were performed using SPSS version 24.0 (SPSS Inc., Chicago, IL, USA).

\section{Results}

Twenty-two eyes of 22 patients (17 males and $5 \mathrm{fe}$ males) who received SRT for treatment of bevacizumabresistant chronic CSC were included in this study based on the inclusion and exclusion criteria. The mean age was $56.3 \pm 8.9$ years (range 36-71 years). All patients were followed up for $\geq 12$ months. All patients had received $\geq 3$ prior intravitreal injections of various anti-VEGF agents, including bevacizumab, ranibizumab, and aflibercept, whereas their last 3 consecutive injections were bevacizumab. Although PDT was 1st recommended for CSC patients, 16 patients received only bevacizumab injections because of the high cost of PDT or the inconvenience caused by light avoidance after PDT. Due to similar reasons, a single injection of ranibizumab or aflibercept was administered to 5 patients prior to the last 3 consecutive bevacizumab injections. The mean number of intravitreal anti-VEGF therapy was $7.2 \pm 6.3$ (range: $3-30)$. The mean duration of persistent SRF was $15.9 \pm$ 7.8 months (range: 6-36 months). The appearance of leakages by FFA examination was classified as focal type in 2 eyes and diffuse type in 20 eyes. All eyes showed PED (4 dome-shaped and 18 flat irregular lesions) on OCT (Table 1). 
Table 1. Baseline demographics and characteristics of 22 eyes of 22 patients with anti-VEGF-resistant chronic CSC

\begin{tabular}{ll}
\hline Patient characteristics & Values \\
\hline Patients (eyes), $n$ & $22(22)$ \\
Age, mean \pm SD, years & $56.3 \pm 8.9$ \\
Sex, $N$ (\%) & Male $17(77.3)$ and female 5 (22.7) \\
Mean duration of current episode, mean \pm SD, months & $15.9 \pm 7.8$ \\
Mean number of intravitreal anti-VEGF therapy prior to SRT, mean \pm SD & $7.2 \pm 6.3$ \\
Type of intravitreal injection used prior to SRT, $N(\%)$ & \\
$\quad$ Bevacizumab & $16(72.7)$ \\
$\quad$ Bevacizumab + ranibizumab & $1(4.5)$ \\
Bevacizumab + aflibercept & $3(13.6)$ \\
$\quad$ Bevacizumab + ranibizumab + aflibercept & $2(9.1)$ \\
Type of leakages, $N$ (\%) & \\
$\quad$ Focal & $2(9.1)$ \\
Diffuse & $20(90.9)$ \\
PED types, $N$ (\%) & \\
$\quad$ Dome shape with various sizes & $4(18.2)$ \\
$\quad$ Flat irregular & $18(81.8)$ \\
BCVA (logMAR), mean \pm SD & $0.49 \pm 0.29$ \\
Central foveal thickness, mean \pm SD, $\mu$ m & $323 \pm 85.6$ \\
SRF height, mean \pm SD, $\mu$ m & $174.6 \pm 86.4$ \\
\hline
\end{tabular}

SD, standard deviation; SRT, selective retina therapy; anti-VEGF, anti-vascular endothelial growth factor; CSC, central serous chorioretinopathy; BCVA, best-corrected visual acuity; logMAR, logarithm of the minimum angle of resolution; PED, pigment epithelial detachment; SRF, subretinal fluid.

Table 2. Changes in BCVA, CMT, and SRF height during the 12-month follow-up period

\begin{tabular}{|c|c|c|c|c|c|c|}
\hline & Baseline & 1 Month & 3 Months & 6 Months & 9 Months & 12 Months \\
\hline \multicolumn{7}{|c|}{ BCVA (logMAR) } \\
\hline Mean & 0.49 & 0.48 & 0.48 & 0.45 & 0.43 & 0.43 \\
\hline SD & 0.29 & 0.32 & 0.32 & 0.33 & 0.39 & 0.36 \\
\hline$p$ value & & 0.704 & 0.858 & 0.189 & 0.168 & 0.067 \\
\hline \multicolumn{7}{|l|}{ CMT, $\mu \mathrm{m}$} \\
\hline Mean & 323 & 251.3 & 252 & 243 & 222.3 & 221.5 \\
\hline $\mathrm{SD}$ & 85.6 & 58.4 & 81.2 & 66.1 & 57.1 & 60.4 \\
\hline$p$ value & & $0.001 *$ & $0.001 *$ & $<0.001^{*}$ & $<0.001^{*}$ & $<0.001^{*}$ \\
\hline \multicolumn{7}{|c|}{ SRF height, $\mu \mathrm{m}$} \\
\hline Mean & 174.6 & 94.7 & 101.4 & 70.2 & 45.5 & 35.1 \\
\hline $\mathrm{SD}$ & 86.4 & 92.0 & 92.4 & 79.4 & 63.6 & 75.4 \\
\hline$p$ value & & $0.001 *$ & $0.001 *$ & $<0.001^{*}$ & $0.001 *$ & $<0.001^{*}$ \\
\hline
\end{tabular}

logMAR, logarithm of the minimal angle of resolution; SRF, subretinal fluid; CMT, central macular thickness; SD, standard deviation; BCVA, best-corrected visual acuity. ${ }^{*} p<0.05$.

The mean BCVA improved from $0.49 \pm 0.29 \log$ MAR at baseline to $0.43 \pm 0.36 \log \mathrm{MAR}$ at 12 months posttreatment $(p=0.067)$. The mean BCVA (logMAR) was $0.06 \log M A R$, equivalent to 3 ETDRS letters. The CMT was significantly decreased from $323 \pm 85.6 \mu \mathrm{m}$ at baseline to $221.5 \pm 60.4 \mu \mathrm{m}$ at 12 months $(p<0.001)$. The mean SRF height decreased significantly from $174.6 \pm$
$86.4 \mu \mathrm{m}$ at baseline to $35.1 \pm 75.4 \mu \mathrm{m}$ at 12 months $(p<$ 0.001) (Table 2).

At 6 months post-treatment, complete and partial responses were observed in 11 (50\%) and 9 (40.9\%) eyes, respectively. Two eyes (9.1\%) showed "no response." At 12 months post-treatment, $18(81.8 \%)$ and $3(13.6 \%)$ eyes showed complete and partial responses, respectively. The 
Fig. 1. Proportion of response based on the rate of resolution of SRF after SRT during a 12-month follow-up period. The decrease of SRF height of $100 \%, \geq 50 \%$, and $<50 \%$ was classified as complete, partial, and no responses, respectively. SRF, subretinal fluid; SRT, selective retina therapy.
Fig. 2. The number of additional SRTs during the 12-month follow-up period. SRTs, selective retina therapies.
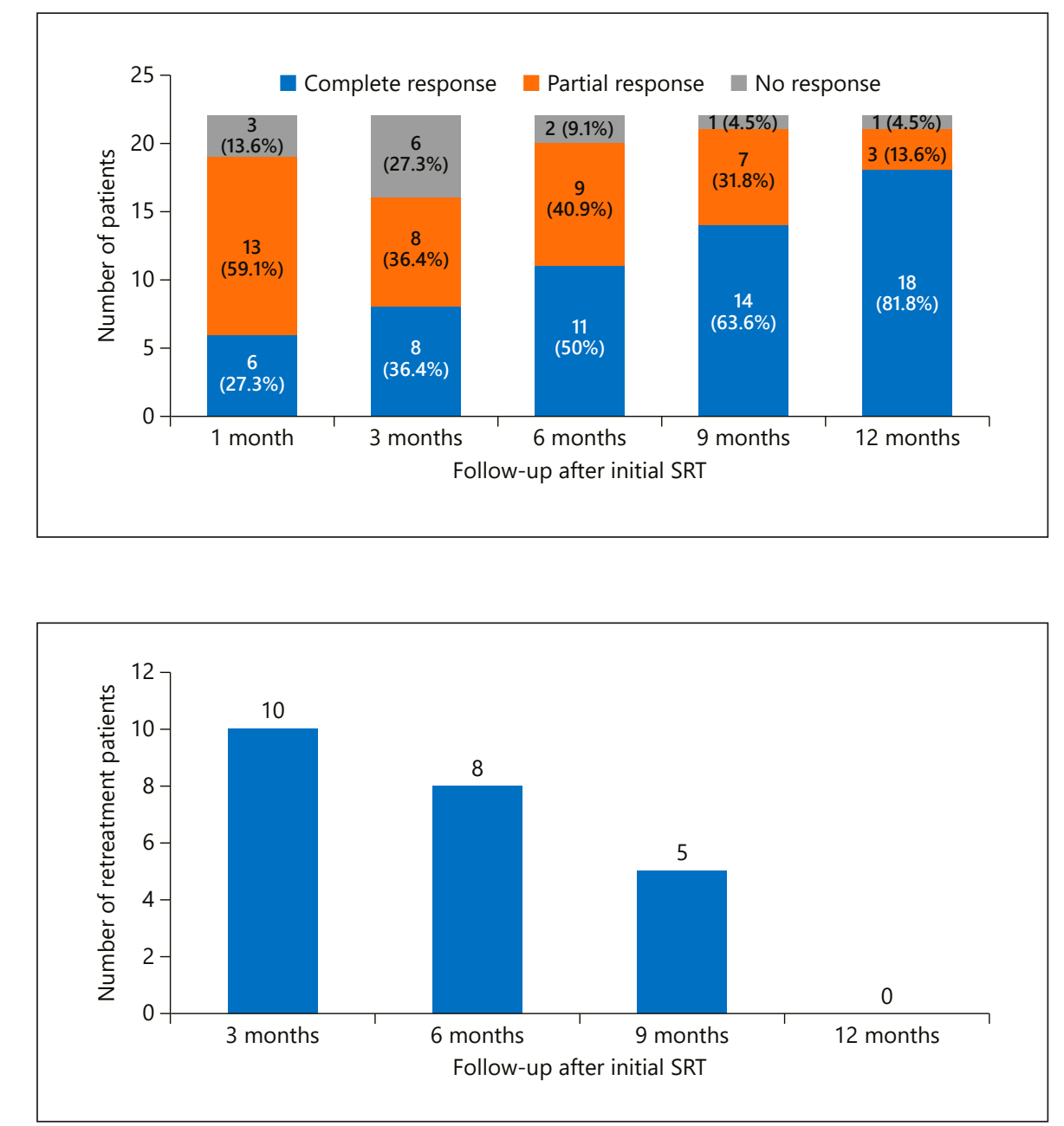

3 eyes showing partial responses experienced complete resolution of SRF during the 12-month follow-up period. Although the remaining 1 (4.5\%) of 22 eyes showed a slight decrease in SRF after the 1st SRT, no response was observed after 3 additional SRT treatments (Fig. 1). The 1 eye showing no response had received 13 anti-VEGF agent injections prior to SRT. Additionally, the eye received a higher mean number of SRT spots (75.6) at each treatment session because it showed diffuse leakage area of $\geq 8$ disc diameters on FFA.

Recurrence was observed in 9 of $22(40.9 \%)$ patients during the 12-month follow-up. Fifteen eyes (68.1\%) required additional SRT due to recurrent or persistent SRF, and the mean number of additional SRT sessions was 1.05 \pm 0.64 (Fig. 2). Eight eyes (36.3\%) underwent an additional SRT session, and 6 eyes (27.2\%) required 2 additional SRT sessions. One eye (4.5\%) required 3 additional SRT procedures.
The mean number of initial treatment spots was 29.9 \pm 22.7 and that of retreatment spots was $30.5 \pm 23.1$. The mean preset pulse energy of the 1st and additional SRT sessions were $124.5 \pm 37.4 \mu \mathrm{J}$ (range: $50-180 \mu \mathrm{J}$ ) and 125.6 $\pm 38.1 \mu \mathrm{J}$ (range: $50-180 \mu \mathrm{J}$ ), respectively.

Although there was no instant discoloration of any treatment SRT spots during irradiation, some barely visible SRT test spots were observed on CFP by $1 \mathrm{~h}$ after irradiation. All barely visible SRT spots became invisible during the 12-month follow-up period (Fig. 3). No hypoautofluorescent changes, indicating RPE atrophy, were observed on the FAF images during the follow-up period. Additionally, no laser-related adverse events, such as retinal hemorrhage and burns, were observed in SRT lesions during the 12-month follow-up period, even after performing 3 additional SRT sessions (Fig. 4). 

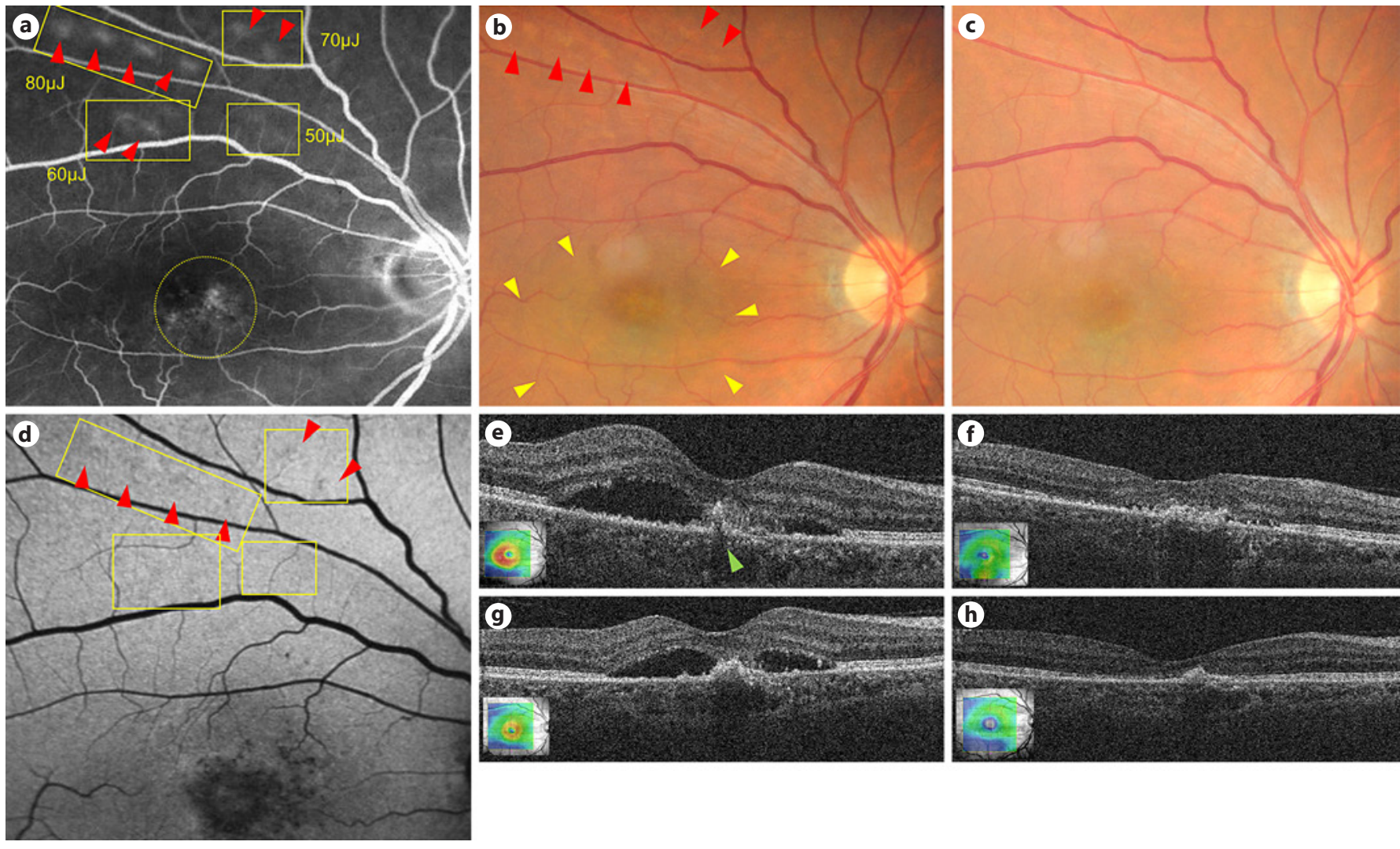

Fig. 3. A 63-year-old male patient with a history of 9 intravitreal bevacizumab injections and 24 months of SRF in the right eye. a The SRT test spots (yellow boxes) comprising 8 spots (4 spots of $80 \mu \mathrm{J}, 2$ spots of $70 \mu \mathrm{J}$, and 2 spots of $60 \mu \mathrm{J}$ ) (red arrowheads) are visible on FFA $1 \mathrm{~h}$ following test spot irradiation. Two spots of 50 $\mu \mathrm{J}$ are invisible on FFA. Although a preset pulse energy of $70 \mu \mathrm{J}$ was initially chosen for treating the area of diffuse leakages on FFA (yellow circle), 27 treatments spots in the range of 90-100 $\mu \mathrm{J}$ were applied according to RFD signals. The spots of $70 \mu \mathrm{J}$ and $80 \mu \mathrm{J}$ show undertreatment alarms (upward-pointing arrows) in the area of SRF, whereas $90 \mu \mathrm{J}$ spots show adequate treatment signals (sideway-pointing arrows). b Six barely visible spots of $70 \mu \mathrm{J}$ and $80 \mu \mathrm{J}$ (red arrowheads) and SRF are observed on fundus photography $1 \mathrm{~h}$ following SRT. c The barely visible spots are invisible on fundus photography at 12 months post-treatment. d Some hyper-

\section{Discussion/Conclusion}

Although anti-VEGF agents have been used for treating patients with CSC, other treatment modalities should be considered if CSC is refractory to repeated intravitreal anti-VEGF injections. As previous studies demonstrated that SRT resulted in favorable functional and anatomical outcomes for acute and chronic CSC [20-25], we have evaluated the efficacy of SRT for bevacizumab-resistant CSC in this study. We demonstrate that SRT was effective autofluorescent pinpoints with hypoautofluorescent semicircle (red arrowheads) are barely visible on FAF at 12 months post-SRT. e SRF with flat irregular PED (green arrowhead) is observed on SD-OCT at the initial presentation. $\mathbf{f}$ The SRF is completely resolved, and hyperreactive materials around flat irregular PED are markedly decreased at 3 months post-SRT. $g$ The second SRT with 28 spots was applied at the same area as the first SRT because of recurrence of SRF at 9 months post-SRT. $h$ Although the SRF is completely resolved, flat irregular PED is still observed on OCT 12 months after the first SRT. SRF, subretinal fluid; SRT, selective retina therapy; FFA, fundus fluorescein angiography; RFD, realtime feedback-controlled dosimetry; PED, pigment epithelial detachment; SD-OCT, spectral-domain optical coherence tomography; OCT, optical coherence tomography; FAF, fundus autofluorescence.
96

Ophthalmologica 2022;245:91-100

DOI: $10.1159 / 000520187$ for improving BCVA and resolving SRF for bevacizumab-resistant CSC during a 12-month follow-up period.

In previous reports, the rate of complete SRF resolution after SRT was reported to be in the range of $71.4-75 \%$ at post-treatment 3 months in patients with chronic CSC $[20,23,24]$. Although the rate of complete SRF resolution at 3 months was $31.8 \%$ in the present study, additional SRT was effective for complete SRF resolution in $81.8 \%$ (18/22 eyes) by 12 months. As $45.5 \%$ (10 of 22 ) of eyes received their 1st additional SRT session at 3 months, re- 

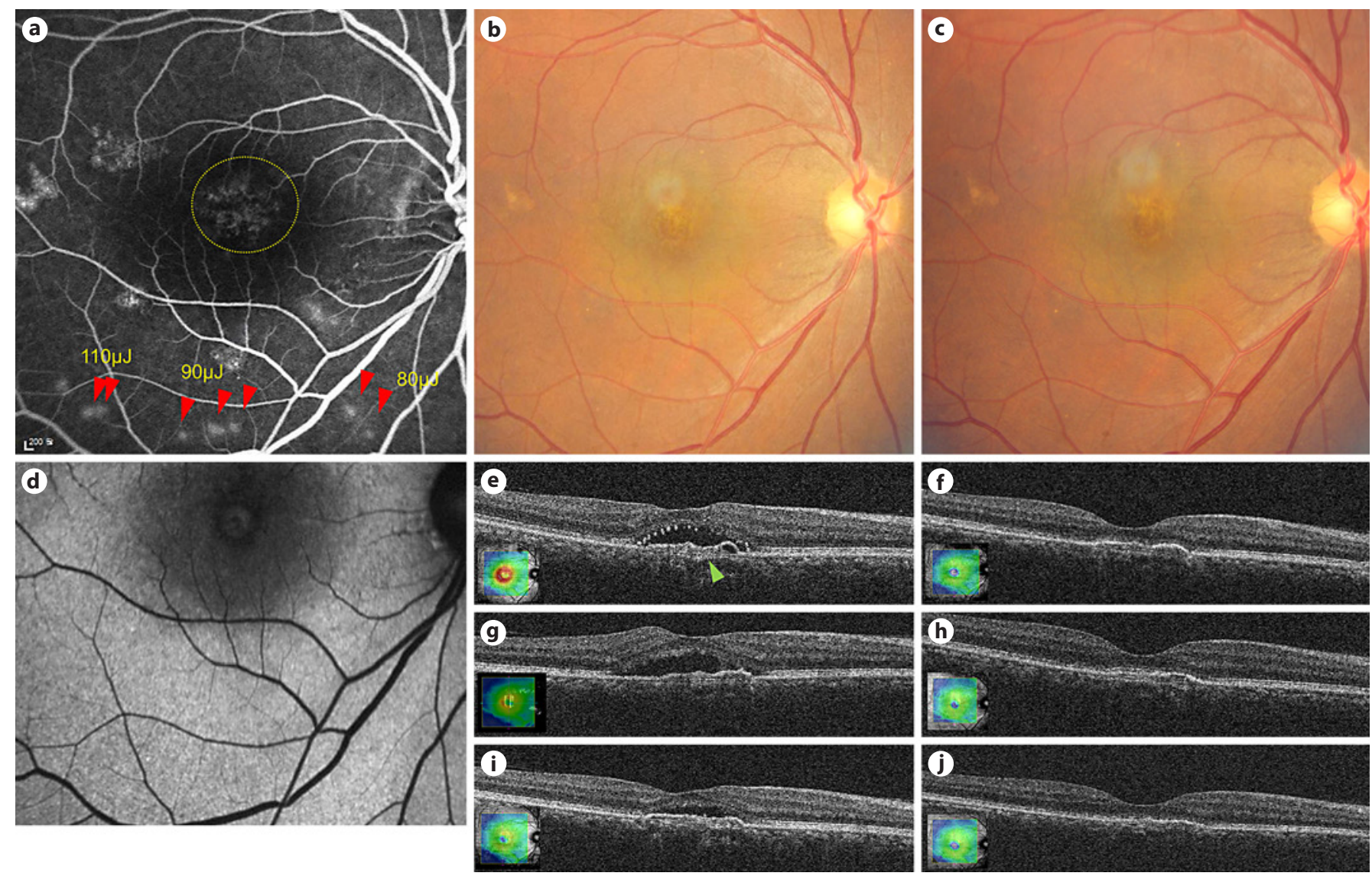

Fig. 4. A 56-year-old male patient with a history of 4 intravitreal bevacizumab injections and SRF persisting for 12 months in the right eye. a Nine SRT test spots of 80, 90, and $110 \mu \mathrm{J}$ (red arrowheads) are visible around the inferior arcade vessel on FFA at $1 \mathrm{~h}$ following test spot irradiation. Nineteen SRT spots were irradiated to cover diffuse leakages (yellow circle) on FFA. b No SRT test spots are visible on fundus photography by $1 \mathrm{~h}$ following test spot irradiation. The demarcation of the SRF is not clear. c All test and treatment SRT spots are invisible on fundus photography 12 months following the first SRT. d SRT test spots are invisible on FAF at 12 months following the first SRT. e SRF with flat irregular

treatment induced a decrease of $31.2 \mu \mathrm{m}$ in the mean SRF height by 6 months. The number of additional SRT sessions at 6 and 9 months decreased from 8 to 5 , as the complete resolution rate gradually increased to $81.8 \%$ by 12 months. However, the SRF resolution rate of patients with bevacizumab-resistant CSC at 3 months was lower than that of patients with CSC in previous studies [21, 23, $25]$. Considering that the mean number of SRT spots using the same irradiation density was reported as 3.8, 9.3, and 21.1 in those previous studies, the number of SRT treatment spots (29.9) in this study suggested that more SRT spots were needed to cover a wider area of diffuse

SRT for Bevacizumab-Resistant Chronic Central Serous Chorioretinopathy
PED (green arrowhead) is observed on SD-OCT at the initial presentation. f SRF is completely resolved at 3 months post-SRT. g The second SRT (20 spots) was performed because of the recurrence of SRF by 6 months following the first SRT. $\mathbf{h}$ SRF is completely resolved at 7 months following the first SRT. i SRT was performed a third time (19 spots) because of recurrence of SRF at 9 months following the first SRT. $\mathbf{j}$ SRF is completely resolved 12 months following the first SRT. SRF, subretinal fluid; SRT, selective retina therapy; FFA, fundus fluorescein angiography; SDOCT, spectral-domain optical coherence tomography; PED, pigment epithelial detachment; FAF, fundus autofluorescence.

leakages. A recent report demonstrated that SRT was more effective in resolving SRF in patients with focal type rather than diffuse type [32]. The complete SRF resolution rate was $74 \%$ when the proportion of diffuse type was $42.9 \%$, whereas the SRF resolution rate was $71.4 \%$ when the proportion of diffuse type was $54 \%$ [20,24]. Although the baseline characteristics of patients were different in previous reports, we speculate that the higher proportion of diffuse type (90.9\%) might negatively influence the response to SRT in this study.

Although the improvement in BCVA was not statistically significant by 12 months, the gain of mean BCVA 
was equivalent to 3 ETDRS letters. This limited improvement in BCVA may be associated with the mean preexisting long-standing SRF (15.9 months) because persistent foveal SRF is known to cause atrophic photoreceptor outer segments, even in the absence of RPE abnormalities [33].

CSC accompanies various types of PED. In acute CSC, dome-shaped focal CSC is common, whereas flat irregular PED is more common in chronic CSC [34]. As flat irregular PED is also a common finding of type $1 \mathrm{CNV}$ in age-related macular degeneration [35], the occurrence of type $1 \mathrm{CNV}$ is thought to be a possible complication of chronic CSC [36]. In a previous study, CNV was observed by OCT angiography in one-third of CSC patients with flat irregular PED [37]. In a recent report, OCT angiography revealed more $\mathrm{CNV}$ in the flat irregular $\mathrm{PED}$ group than in the focal PED group (42.9\% vs. 10.0\%). Based on the presence or absence of CNV on OCT angiography, responsiveness to anti-VEGF injection was significantly higher in eyes with CNV than in eyes without CNV $(100 \%$ vs. 33.3\%) [38]. Although OCT angiography was not used in the current study, we speculate that there is little possibility of the presence of concomitant CNV in bevacizumab-resistant CSC patients.

Flat irregular PED was known to be associated with pachychoroid disease; however, RPE-targeting SRT was effective in resolving SRF in the current study. We suspect that bioactive substances during RPE restoration might play a role to resolve SRF [39]. Additionally, the effect of SRT is known to be associated with the restoration of the outer blood-retinal barrier in terms of new RPE restoration [19]. FAF was used to evaluate the SRT lesions during 1-year follow-up visits; however, no dark black change indicating RPE atrophy was observed on FAF in the present study.

In this study, the mean preset pulse energy $(134.8 \mu \mathrm{J})$ for treatment spots was higher than that $(116.3 \mu \mathrm{J})$ of previous report [24]. Since the higher preset pulse energy could be needed to achieve adequate SRT lesions in the area with SRF, the higher mean SRF height $(174.6 \mu \mathrm{m})$ than that of the previous study $(168.0 \mu \mathrm{m})$ may have influenced the increase of preset pulse energy. Since the minimum preset pulse energy was chosen based on the response of the test spot in the healthy area, the initial treatment spots for the diseased area with SRF showed frequent undertreatment alarms (upward-pointing arrows) by the RFD. Therefore, if the range of preset pulse energy based on test spots is within the range of adequate RPE damage, we suggest that the initial preset pulse energy for the area of SRF could be chosen from higher pre- set energy values, rather than from the minimum preset energy, indicating FFA-positive test spots in CSC patients with a greater SRF height. In addition, no SRT-related adverse events, including retinal hemorrhage and burns, indicating overtreatment, were observed in the current study.

Other laser modalities such as subthreshold micropulse laser (SMPL) and PDT have been used for CSC. In the PLACE trial, the half-dose PDT group showed the higher complete SRF resolution rate (67\%) than the SMPL group (29\%) 6-7 months following treatment [40]. In the current study, $50 \%$ of patients showed complete SRT resolution by 6 months. Unlike SRT, SMPL does not produce any RPE damage during the process of therapeutic action. Besides the difference of tissue response after each laser, the therapeutic mechanism of SMPL is thought to be different from that of SRT [41]. Therefore, our result could not be applied to the effect of other subthreshold laser modalities.

Our study had several limitations. First, a retrospective and nonrandomized study design was used, and small case numbers were included. Second, although all the patients were treated with 3 consecutive bevacizumab injections prior to SRT, other kinds of anti-VEGF agents could have been considered for treating bevacizumab-resistant CSC. Third, since CSC is known as a pachychoroid disease, the change of choroidal thickness could be helpful to evaluate the effect of SRT. However, the choroidal thickness data were not included in the current study since enhanced depth images were available in some patients. Fourth, although OCT angiography can reveal CNV, which was not detected by FFA or ICG [38], OCT angiography was not available in the present study. Considering that anti-VEGF agent was more effective for CSC with CNV than for CSC without $\mathrm{CNV}$, bevacizumab-resistant CSC has little possibility of the presence of concomitant CNV. CSC with flat irregular PED could be a sign of quiescent CNV per se, or it could progress to CNV [42]. However, there was no retinal exudation or hemorrhage on multimodal images, indicating $\mathrm{CNV}$, during the 12-month follow-up in the present study.

In our study, we demonstrated that SRT with RFD was effective in resolving SRF in patients with bevacizumabresistant CSC. Although the response to SRT was relatively low at 3 months, additional SRT sessions reinforced the resolution of SRF during the 12-month follow-up. Since no SRT-related adverse events were observed following multiple sessions of SRT, RFD was useful in avoiding overtreatment. Since SRT was performed based on
98

Ophthalmologica 2022;245:91-100 DOI: $10.1159 / 000520187$
Jeon/Kim/Lee/Roh 
the preset pulse energy provided by pretreatment FFA in this study, FFA remains a mandatory method to confirm RPE damage during the SRT procedure. Further studies are warranted for elucidating the use of RFD as a substitute for FFA.

SRT showed favorable visual and anatomical longterm outcomes in patients with bevacizumab-resistant chronic CSC. Larger randomized studies are warranted to determine the effect of SRT as a treatment option for anti-VEGF-resistant chronic CSC.

\section{Statement of Ethics}

This retrospective study adhered to the principles of the 1964 Declaration of Helsinki, and data collection was compliant with and approved by the Institutional Review Board of Yeouido St Mary's Hospital of the Catholic University of Korea. Written informed consent was obtained from all patients in this study after having been told about possible risks of SRT treatment.

\section{Conflict of Interest Statement}

Young-Jung Roh has a patent related to real-time feedback dosimetry in South Korea (10-2018-004956). The SRT laser system (R:Gen; Lutronic) was approved by the Ministry of Food and Drug
Safety in South Korea for patients with central serous chorioretinopathy. Lutronic provided the SRT laser system and technical support but had no role in the study design.

\section{Funding Sources}

This study was supported by a grant of the Institute of Clinical Medicine Research in the Yeouido St. Mary's Hospital, Catholic University of Korea.

\section{Author Contributions}

Young-Jung Roh conceived and designed the experiments; Young-Jung Roh performed the treatment; Seung Hee Jeon, Minhee Kim, Jiyoung Lee, and Young-Jung Roh analyzed the data; Seung Hee Jeon, Minhee Kim, and Jiyoung Lee contributed reagents/materials/analysis tools; Seung Hee Jeon, Minhee Kim, Jiyoung Lee, and Young-Jung Roh wrote the paper; Seung Hee Jeon, Minhee Kim, Jiyoung Lee, and Young-Jung Roh approved the final version of the manuscript.

\section{Data Availability Statement}

All data generated or analyzed during this study are included in this article. Further inquiries can be directed to the corresponding author.

\section{References}

1 Wang M, Munch IC, Hasler PW, Prünte C, Larsen M. Central serous chorioretinopathy. Acta Ophthalmol. 2008;86:126-45.

2 Kitzmann AS, Pulido JS, Diehl NN, Hodge DO, Burke JP. The incidence of central serous chorioretinopathy in Olmsted County, Minnesota, 1980-2002. Ophthalmology. 2008; 115:169-73.

3 Piccolino FC, Borgia L, Zinicola E, Zingirian $M$. Indocyanine green angiographic findings in central serous chorioretinopathy. Eye. 1995;9 (Pt 3):324-32.

4 Guyer DR, Yannuzzi LA, Slakter JS, Sorenson JA, Ho A, Orlock D. Digital indocyanine green videoangiography of central serous chorioretinopathy. Arch Ophthalmol. 1994; 112:1057-62.

5 Sulzbacher F, Schütze C, Burgmüller M, Vécsei-Marlovits PV, Weingessel B. Clinical evaluation of neovascular and non-neovascular chronic central serous chorioretinopathy (CSC) diagnosed by swept source optical coherence tomography angiography (SS OCTA). Graefes Arch Clin Exp Ophthalmol. 2019;257:1581-90.

6 Gass JD. Pathogenesis of disciform detachment of the neuroepithelium. Am J Ophthalmol. 1967;63:Suppl 1-139.
7 Laatikainen L. Diffuse chronic retinal pigment epitheliopathy and exudative retinal detachment. Acta Ophthalmol. 1994;72:533-6.

8 Daruich A, Matet A, Dirani A, Bousquet E, Zhao M, Farman N, et al. Central serous chorioretinopathy: recent findings and new physiopathology hypothesis. Prog Retin Eye Res. 2015;48:82-118.

9 Klein ML, Van Buskirk EM, Friedman E, Gragoudas E, Chandra S. Experience with nontreatment of central serous choroidopathy. Arch Ophthalmol. 1974;91:247-50.

10 Mrejen S, Balaratnasingam C, Kaden TR, Bottini A, Dansingani K, Bhavsar KV, et al. Longterm visual outcomes and causes of vision loss in chronic central serous chorioretinopathy. Ophthalmology. 2019;126:576-88.

11 Loo RH, Scott IU, Flynn HW Jr, Gass JD, Murray TG, Lewis ML, et al. Factors associated with reduced visual acuity during longterm follow-up of patients with idiopathic central serous chorioretinopathy. Retina. 2002;22:19-24.

12 van Rijssen TJ, van Dijk EHC, Yzer S, OhnoMatsui K, Keunen JEE, Schlingemann RO, et al. Central serous chorioretinopathy: towards an evidence-based treatment guideline. Prog Retin Eye Res. 2019;73:100770.
13 Ficker L, Vafidis G, While A, Leaver P. Longterm follow-up of a prospective trial of argon laser photocoagulation in the treatment of central serous retinopathy. Br J Ophthalmol. 1988;72:829-34.

14 Khosla PK, Rana SS, Tewari HK, Azad RU, Talwar D. Evaluation of visual function following argon laser photocoagulation in central serous retinopathy. Ophthalmic Surg Lasers. 1997;28:693-7.

15 Huang WC, Chen WL, Tsai YY, Chiang CC, Lin JM. Intravitreal bevacizumab for treatment of chronic central serous chorioretinopathy. Eye. 2009;23:488-9.

16 Schaal KB, Hoeh AE, Scheuerle A, Schuett F, Dithmar S. Intravitreal bevacizumab for treatment of chronic central serous chorioretinopathy. Eur J Ophthalmol. 2009;19:613-7.

17 Ruiz-Moreno JM, Lugo FL, Armadá F, Silva R, Montero JA, Arevalo JF, et al. Photodynamic therapy for chronic central serous chorioretinopathy. Acta Ophthalmol. 2010;88: 371-6.

18 Lim JI, Glassman AR, Aiello LP, Chakravarthy U, Flaxel CJ, Spaide RF. Collaborative retrospective macula society study of photodynamic therapy for chronic central serous chorioretinopathy. Ophthalmology. 2014;121:1073-8. 
19 Brinkmann R, Roider J, Birngruber R. Selective retina therapy (SRT): a review on methods, techniques, preclinical and first clinical results. Bull Soc Belge Ophtalmol. 2006;(302): 51-69.

20 Klatt C, Saeger M, Oppermann T, Pörksen E, Treumer F, Hillenkamp J, et al. Selective retina therapy for acute central serous chorioretinopathy. Br J Ophthalmol. 2011;95:83-8.

21 Elsner H, Pörksen E, Klatt C, Bunse A, Theisen-Kunde D, Brinkmann R, et al. Selective retina therapy in patients with central serous chorioretinopathy. Graefes Arch Clin Exp Ophthalmol. 2006;244:1638-45.

22 Framme C, Walter A, Berger L, Prahs P, Alt $\mathrm{C}$, Theisen-Kunde $\mathrm{D}$, et al. Selective retina therapy in acute and chronic-recurrent central serous chorioretinopathy. Ophthalmologica. 2015;234:177-88.

23 Kang S, Park YG, Kim JR, Seifert E, TheisenKunde D, Brinkmann R, et al. Selective retina therapy in patients with chronic central serous chorioretinopathy: a pilot study. Medicine. 2016;95:e2524.

24 Park YG, Kang S, Kim M, Yoo N, Roh YJ. Selective retina therapy with automatic realtime feedback-controlled dosimetry for chronic central serous chorioretinopathy in Korean patients. Graefes Arch Clin Exp Ophthalmol. 2017;255:1375-83.

25 Büttner M, Luger B, Luger B, Abou Moulig W, Junker B, Framme C, et al. Selective retina therapy (SRT) in patients with therapy refractory persistent acute central serous chorioretinopathy (CSC): 3 months functional and morphological results. Graefes Arch Clin Exp Ophthalmol. 2021;259(6):1401-10.

26 Schuele G, Elsner H, Framme C, Roider J, Birngruber R, Brinkmann R. Optoacoustic real-time dosimetry for selective retina treatment. J Biomed Opt. 2005;10:064022.
27 Seifert E, Tode J, Pielen A, Theisen-Kunde D, Framme C, Roider J, et al. Selective retina therapy: toward an optically controlled automatic dosing. J Biomed Opt. 2018;23:1-12.

28 Park YG, Seifert E, Roh YJ, Theisen-Kunde D, Kang S, Brinkmann R. Tissue response of selective retina therapy by means of a feedbackcontrolled energy ramping mode. Clin Exp Ophthalmol. 2014;42:846-55.

29 Kim HD, Jang SY, Lee SH, Kim YS, Ohn YH, Brinkmann R, et al. Retinal pigment epithelium responses to selective retina therapy in mouse eyes. Invest Ophthalmol Vis Sci. 2016; 57:3486-95.

30 Minhee K, Park YG, Kang S, Roh YJ. Comparison of the tissue response of selective retina therapy with or without real-time feedback-controlled dosimetry. Graefes Arch Clin Exp Ophthalmol. 2018;256:1639-51.

31 Kim M, Park YG, Roh YJ. One-year functional and anatomical outcomes after selective retina therapy with real-time feedback-controlled dosimetry in patients with intermediate age-related macular degeneration: a pilot study. Lasers Surg Med. 2021;53(4):499-513.

32 Kyo A, Yamamoto M, Hirayama K, Kohno T, Theisen-Kunde D, Brinkmann R, et al. Factors affecting resolution of subretinal fluid after selective retina therapy for central serous chorioretinopathy. Sci Rep. 2021;11(1):8973.

33 Wang M, Sander B, la Cour M, Larsen M. Clinical characteristics of subretinal deposits in central serous chorioretinopathy. Acta Ophthalmol Scand. 2005;83:691-6.

34 Song IS, Shin YU, Lee BR. Time-periodic characteristics in the morphology of idiopathic central serous chorioretinopathy evaluated by volume scan using spectral-domain optical coherence tomography. Am J Ophthalmol. 2012;154:366-75.e4.

35 Keane PA, Patel PJ, Liakopoulos S, Heussen FM, Sadda SR, Tufail A. Evaluation of agerelated macular degeneration with optical coherence tomography. Surv Ophthalmol. 2012;57:389-414.
36 Hage R, Mrejen S, Krivosic V, Quentel G, Tadayoni R, Gaudric A. Flat irregular retinal pigment epithelium detachments in chronic central serous chorioretinopathy and choroidal neovascularization. Am J Ophthalmol. 2015;159:890-903.e3.

37 Bousquet E, Bonnin S, Mrejen S, Krivosic V, Tadayoni R, Gaudric A. Optical coherence tomography angiography of flat irregular pigment epithelium detachment in chronic central serous chorioretinopathy. Retina. 2018; 38(3):629-38.

38 Hwang H, Kim JY, Kim KT, Chae JB, Kim DY Flat irregular pigment epithelium detachment in central serous chorioretinopathy: a form of pachychoroid neovasculopathy? Retina. 2020;40:1724-33.

39 Richert E, Koinzer S, Tode J, Schlott K, Brinkmann R, Hillenkamp J, et al. Release of different cell mediators during retinal pigment epithelium regeneration following selective retina therapy. Invest Ophthalmol Vis Sci. 2018; 59:1323-31.

40 van Dijk EHC, Fauser S, Breukink MB, Blanco-Garavito R, Groenewoud JMM, Keunen JEE, et al. Half-dose photodynamic therapy versus high-density subthreshold micropulse laser treatment in patients with chronic central serous chorioretinopathy: the PLACE trial. Ophthalmology. 2018;125:1547-55.

41 Chhablani J, Roh YJ, Jobling AI, Fletcher EL, Lek JJ, Bansal P, et al. Restorative retinal laser therapy: present state and future directions. Surv Ophthalmol. 2018;63:307-28.

42 Querques G, Srour M, Massamba N, Georges A, Ben Moussa N, Rafaeli O, et al. Functional characterization and multimodal imaging of treatment-naive "quiescent" choroidal neovascularization. Invest Ophthalmol Vis Sci. 2013;54:6886-92. 